\title{
Design of a study evaluating the effects, health economics, and stakeholder perspectives of a multi-component occupational rehabilitation program with an added workplace intervention - a study protocol
}

Marit B. Rise ${ }^{1,2^{*}}$ D, Martin Skagseth ${ }^{1}$, Nina E. Klevanger ${ }^{1}$, Lene Aasdahl ${ }^{1,3}$, Petter Borchgrevink ${ }^{4,5}$, Chris Jensen ${ }^{1,6}$, Hanne Tenggren ${ }^{4}$, Vidar Halsteinli ${ }^{1}$, Trym N. Jacobsen ${ }^{3,4}$, Svein B. Løland ${ }^{4}$, Roar Johnsen ${ }^{1}$ and Marius S. Fimland ${ }^{1,4}$

\begin{abstract}
Background: Recent research has suggested that interventions at the workplace might be the most potent ingredient in return to work interventions, but few studies have investigated the different effects of workplace interventions as part of occupational rehabilitation programs. The comprehensive design described in this article includes effect (on return to work and health outcomes), and health economic evaluations of a workplace intervention added to a multicomponent rehabilitation program. Qualitative and mixed method studies will investigate sick-listed persons', rehabilitation therapists' and employers' perspectives on the usability and outcomes of the rehabilitation program and the workplace intervention. The program and intervention are provided to patients with musculoskeletal, psychological or general and unspecified diagnoses. The program is multi-component and includes Acceptance and Commitment Therapy, physical exercise, patient education and creating a plan for increased work participation.

Methods: Persons who are employed, aged from 18 to 60 years, with a current sick leave status of 50\% or more and a diagnosis within the musculoskeletal, psychological or general and unspecified chapters of International Classification of Primary Care-2 (ICPC-2) will be recruited to a researcher-blinded parallel-group randomized controlled trial. All participants take part in an in-patient occupational rehabilitation program, while the intervention group also takes part in an intervention at the workplace. The effect and economic evaluation will investigate the effect of the added workplace intervention. The primary outcome measures will be time until full sustainable return to work and total number of sickness absence days in the 12 months after inclusion. Health economic evaluations will investigate the cost-effectiveness and cost-utility. Qualitative studies will investigate rehabilitation therapists' experiences with working towards return to work within an ACT-approach and stakeholders' experiences with the workplace intervention. A mixed methods study will combine quantitative and qualitative findings on the participants' expectations and motivation for return to work.

(Continued on next page)
\end{abstract}

\footnotetext{
* Correspondence: marit.b.rise@ntnu.no

'Department of Public Health and Nursing, Norwegian University of Science and Technology, Trondheim, Norway

${ }^{2}$ Department of Mental Health, Norwegian University of Science and

Technology, 7491 Trondheim, Norway

Full list of author information is available at the end of the article
} 
(Continued from previous page)

Discussion: The outline of this comprehensive study could represent an important addition to the standard designs of return to work evaluation. The mixed methods design, with qualitative approaches as well as a rigorous randomized controlled trial, might prove useful to shed light on contextual factors.

Trial registration: ClinicalTrials.gov: NCT02541890. September 4, 2015.

Keywords: Absenteeism, Occupational health, Mental health, Return to work, Acceptance and commitment therapy, Cognitive behaviour therapy, Musculoskeletal diseases

\section{Background}

Work disability is considered one of the biggest challenges for governments and policy makers in The organisation for economic co-operation and development (OECD) countries [1]. In Norway, sickness absence rates are the highest in the OECD area [2], with costs of disability benefits totaling to approximately $4-5 \%$ of GDP in 2010 [1]. Hence, many European countries, including Norway, have implemented legislation and acts to prevent long-term absence and work disability due to health problems [3], and many rehabilitation programs have been established to help patients return to work (RTW) [3-9]. In Norway, inpatient occupational rehabilitation programs are widespread. They usually consist of group-based and individual cognitive behavioral- and physical exercise sessions, as well as patient education [10-12], but have rarely included workplace interventions [13]. Individuals are usually referred to such programs by their general practitioner, but can also be referred from the Welfare and Labor Service.

So far, most occupational rehabilitation programs have been tailored for specific diagnosis groups, most often for musculoskeletal disorders [7, 14-17] and some for mental health problems [18-21]. Several of these programs have shown effect on return to work and other outcomes [7, 14]. Different models have been used to help understand work disability; the medical model, the biopsychosocial model, and the case management ecological model, where the environmental influence has gradually become more important. Now, political, economic, cultural and work place environment are considered to interact with the persons own behavior and attitudes [22]. Thus, sickness absence is no longer solely attributed to the person's health problems, but is considered a result of complex interactions between the person's physical and behavioral characteristics and the socio-cultural environment, including the health services, the work environment and the system providing financial compensation [22, 23]. Together with an increased focus on the high level of co-morbidity among sick-listed persons [24-27], these developments warrant more generic programs in order to be useful for patients with different and overlapping diagnoses. The effect of generic programs are still unclear. In addition, many have argued that interventions to prevent work disability and long-term sickness-absence should address all stakeholders and systems involved in this field [28, 29]. Employers have a key role in the return to work process [1] that seems to be confirmed in systematic literature reviews [30-32]. Similarly, the need for collaboration between different types of health care professionals in managing and preventing disability has also been highlighted in the literature [16, 22, 33]. Collaboration and coordination between the different actors is thus needed to prevent prolonged sickness absence and to improve return to work [22, 34].

Workplace interventions are defined as initiatives linked to the workplace, including adaptations at work or involvement of the work-environment stakeholders. A landmark study from Canada suggested that interventions at the workplace might be the most potent ingredient in occupational rehabilitation, especially when combined with a clinical intervention [16]. The study was later partly replicated in the Netherlands and Denmark [7, 35, 36]. In a Dutch study Lambeek et al. [7] found that a workplace intervention as part of a clinical rehabilitation program was more effective in facilitating return to work than usual care. However, the effects of specific intervention components are uncertain and publication bias is present [37]. Several systematic reviews have reported that, for patients with back pain, and to some extent for patients with mental disorders, multimodal rehabilitation which includes interventions at the workplace is more effective than other interventions [30-32, 38]. However, the effect of workplace interventions on sustainable return to work is so far unclear [32]. It seems important to involve the different actors, such as patient and employer, in active consultation and consensus about how to modify the work environment, specific work tasks or the work organization to facilitate return to work [38,39], and this collaborative approach is recommended [29].

Hence, current knowledge points towards more multimodal and generic occupational rehabilitation programs that comprise the different stakeholders in the return to work process, including the workplace. There is thus a need for effect studies which can attend to the complexity of multimodal interventions, as well as more heterogeneous patient groups. In addition to randomized controlled designs to investigate effects on return to work, qualitative methodological approaches should focus on the 
experiences of the different stakeholders in the return to work process (sick listed persons, service providers, and employers), and help uncover any unknown facilitators and barriers in the return to work process. Qualitative methodological approaches could also investigate the stakeholders' perceptions of usability and other potential outcomes from initiatives to support work participation and return to work. It is also necessary to assess the cost-benefit of such interventions through economic evaluations.

\section{Objectives}

The objective of this article is to describe the protocol of a study which endeavors to encompass all the requirements described above. The proposed study design includes an effect evaluation (a randomized controlled trial), an economic evaluation, two qualitative studies and one mixed methods study which combines qualitative and quantitative data. The proposed study design is presented in Fig. 1.

The development of this study and the study design is based on the experiences from a large randomized controlled trial (RCT) conducted in Central Norway $[11,12,40]$. Interviews with rehabilitation therapists and participants made it clear that very few participants had been in contact with the workplace or employer during the rehabilitation program. A qualitative study also showed that the participants, at the end of the program, perceived return to work as a long and complex process, and few of them felt ready to return to work [41]. To enhance the return to work focus and improve the return to work rates, the researchers, in collaboration with the clinic, developed and piloted an intervention at the participants' workplace. This, added to the rehabilitation program, is proposed as the intervention in the RCT.

Based on the findings of high comorbidity in sick-listed persons, and to encompass the largest group of sick-listed persons in Norway, the rehabilitation programs and the workplace intervention are developed specifically for a heterogeneous patient group; patients with a diagnosis within the musculoskeletal, psychological or general and unspecified chapters of International Classification of Primary Care-2 (ICPC-2). Large part of the program's psychological therapy is provided in groups where persons with different diagnoses are mixed. This is still a rather new approach in occupational rehabilitation. The therapists' educational backgrounds are also heterogeneous, including backgrounds such as nurses, physiotherapists, psychologists, and social workers. All were provided training and supervision in the same psychological therapy approach. Acceptance and Commitment Therapy (ACT) [42, 43] is chosen as the psychological therapy approach. The ACT approach has, so far, very limited use in occupational therapy $[40,44,45]$.

\section{The Norwegian sickness absence system}

All legal residents in Norway are included in the Norwegian public insurance system. Medically certified sick leave is

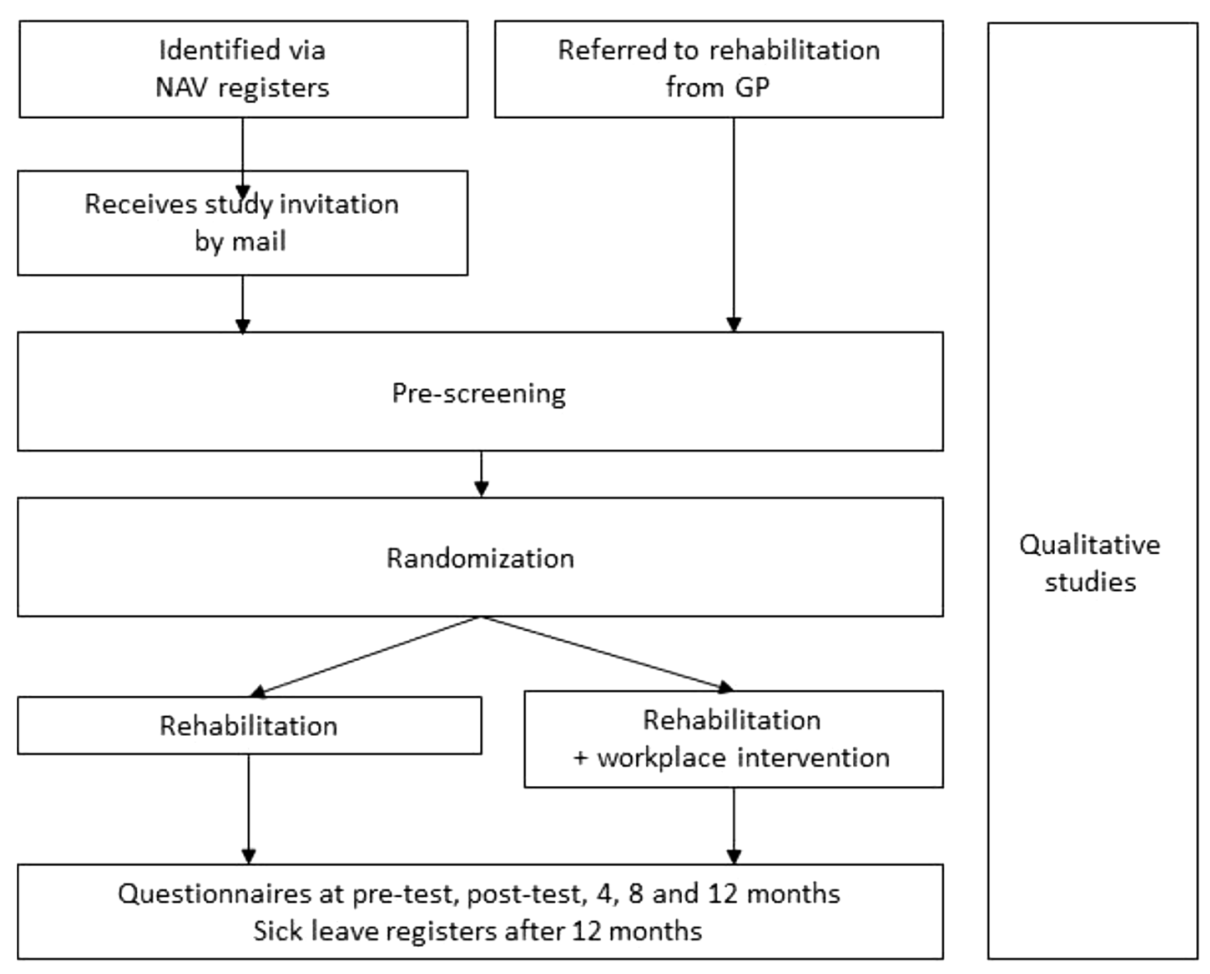

Fig. 1 Study design 
compensated with $100 \%$ coverage for the first 12 months. The medical certificate can, and is encouraged if possible, to be graded from $20 \%$ to $100 \%$, independent of employment fraction. This means that if you are employed in 50\% position and are $50 \%$ on sick leave, you are working $25 \%$. After 12 months of sick leave, it is possible to apply for long-term medical benefits, compensating approximately $66 \%$ of the income.

\section{Methods}

The aims of the studies are to:

1) Through a randomized controlled trial, investigate whether an occupational rehabilitation program with a workplace intervention can improve sustainable return to work and reduce sickness absence, compared to an occupational rehabilitation program without a workplace intervention.

2) Through an economic evaluation, assess whether an occupational rehabilitation program with a workplace intervention is cost effective, compared to a program without a workplace intervention.

3) Through two qualitative studies and one mixed methods study, investigate the patients, rehabilitation therapists, and employers' experiences of the usability and perceived outcomes of the programs and the intervention.

\section{Participants and recruitment}

People living in Trøndelag (two counties in Central Norway) are eligible for inclusion if they 1 ) are aged 18 to 60 years, 2) are sick-listed 2-12 months, 3) hold at least a $20 \%$ job position (e.g. minimum one day per week), 4) have an employer, 5) have a sick-leave status of at least $50 \%$ off work, 6) anticipate at least four more weeks of sick-leave, and 7) have a diagnosis within the musculoskeletal, psychological or general and unspecified chapters of ICPC-2. The participants' diagnoses are as assessed by the participants' general practitioner (GP). In addition, potential participants must currently have an employer. Exclusion criteria are: Being selfemployed, having or being under consideration for a serious somatic or mental health/substance abuse disorder (assessed by the screening clinic), currently undergoing rehabilitation, having significant problems with working in a group (assessed by the screening clinic based on questionnaires and clinical interview), insufficient comprehension of Norwegian language to participate in group sessions and to fill out questionnaires, scheduled for surgery within the next 6 months, or being pregnant.

\section{Sample size}

Sample size is calculated in line with the requirements in the randomized controlled trial. Comparison of return to work with Kaplan Meier survival analysis with log rank test with a hazard ratio of 0.6 (alpha 0.05, beta 0.20 ) would require 63 participants in each group. As we use register-based sickness absence data, there will be no loss to follow-up in the intention-to-treat analyses of primary outcomes. However, to provide greater statistical power for questionnaire-based outcomes we will include at least 80 participants in each group. The sample size estimation was based on results from previous studies in this field $[7,16,36]$, as well as preliminary results from ongoing studies [11].

\section{Recruitment to the effect evaluation and economic evaluation} Potential participants are recruited in two ways. 1) The Norwegian Labour and Welfare Service (NAV) will provide monthly lists of persons meeting the inclusion criteria. An assigned project co-worker sends out invitations to potential participants who accept to participate by contacting the research group. Persons who accept to participate are invited to an out-patient screening clinic for clinical examination and interview before inclusion. 2) General practitioners (GPs) refer patients to the study and the rehabilitation program. The GPs will be provided with information of the project through written leaflets, the website of the rehabilitation centre (www.stolav.no/hysne shelsefort), and can call a project co-worker for any questions. The GPs will refer potential participants directly to the out-patient screening clinic for clinical examination and interview. Besides the project co-worker who sends out the invitation letters, none of the researchers in the project will know the identity of potential participant, until the person accepts to participate through answering the invitation letter or accepts to being referred to the program by their GP.

All participants will complete baseline study outcome measurements and several screening questionnaires before an out-patient screening is performed. The screening is conducted by a medical doctor, a psychologist and physiotherapist during clinical examination and interview. The clinic assesses the final eligibility before randomization and excludes all persons who do not fit the inclusion criteria. The personnel at the screening clinic will also give oral and written information about the study, and emphasize that agreeing to participate includes being randomized to a program with or without a workplace intervention. All participants who are included by the screening clinic will sign a written consent form prior to randomization. Taking part in the RCT will be a prerequisite for participation in the rehabilitation program and the intervention. The participants can decline participation and withdraw from the study (and program) at any point. 


\section{Randomization}

Persons who are found eligible and sign an informed consent will be randomized to the intervention arm (rehabilitation program with a workplace intervention) or the control arm (rehabilitation program only). Block randomization with unknown block sizes is performed using a web-based program delivered by a third party the Unit for Applied Clinical Research at the Norwegian University of Science and Technology.

\section{Recruitment to qualitative and mixed methods studies}

All participants in the RCT will be eligible to take part in qualitative studies. Participants will be recruited strategically to inform the specific research aims. Potential participants will be given written and oral information about the studies and all participants will sign a consent form before taking part in interviews or observation. Potential participants in the first of the two qualitative studies are all rehabilitation therapists at the center, including all those providing the workplace intervention. They will be contacted directly by the researchers, and we aim to recruit 10-15 therapists. For the observation in the second qualitative study, all participants in one program group will receive written information and asked to consent to having a researcher present in their group during the rehabilitation program. All participants in the group must consent to the observation. Potential participants in the second qualitative study are the participants in the intervention arm, their employers and rehabilitation therapists. First, the participant will be given written information, and asked to take part in interviews and whether a researcher can observe the workplace intervention. If the participant consents, their employer will be contacted with the same information. The rehabilitation therapist will also be asked by the researcher to take part in interviews. We aim to recruit a sample where the participants' gender, type of occupation, age, and length of sickness absence is varied. We aim to recruit 1015 triads (participant, therapist and employer). Potential participants in the mixed methods study are the participants (from intervention group and control group) who have completed questionnaires 4 months after the end of the rehabilitation program. A sample of approximately 20 of these participants will be asked to take part in individual interviews. This will be a strategic sample to explore their perspectives on the return to work process in interviews four months after the rehabilitation programs, and to elaborate on quantitative data already collected in the RCT on the same subject.

\section{The occupational rehabilitation program}

Regardless of group allocation in the RCT, all study participants take part in an occupational rehabilitation program. The program is composed of elements that are assumed to be useful in occupational rehabilitation for long-term sickness, and includes physical exercise, psychological therapy, and work related problem solving. Hysnes Rehabilitation Center (www.stolav.no/hysneshelse fort) is the setting for the occupational rehabilitation programs. The center was established as part of St. Olav's Hospital and is located in the municipality of Rissa, a $50 \mathrm{~min}$ boat ride or $90 \mathrm{~min}$ drive from Trondheim, the third largest city (181.500 inhabitants) in Norway. The center has provided in-patient occupational rehabilitation programs since 2010.

The rehabilitation program lasts four weeks; the first two weeks at the rehabilitation center, the third week at home, and the fourth week at the center, engaging participants in full 'work' days, aiming to establish normal daily routines. The program is provided by interdisciplinary teams consisting of psychologists, physiotherapists, exercise physiologists, nurses, physician and welfare case worker provide the programs. All professionals are trained as ACT therapists. Each therapist treats 2-4 clients individually and in groups, focusing on behavior change and coping. Two therapists are assigned to each group which includes 6-8 participants. A mid-way report and a final report are sent to the GP. If the participant agrees, the reports are also sent to the participant's welfare office and employer.

\section{Physical exercise}

Physical exercise is utilized to improve health, fitness, functional capacities, and to reduce fear-avoidance related to physical activity. An exercise plan is made for each participant and follow-up is provided during the program, both in groups and individually. The main objective for individual follow-up of exercise during the program is to ensure that the exercise plan is suitable and realistic and can be useful for the participant after the program.

\section{Acceptance and commitment therapy (ACT)}

The psychological therapy approach is ACT, an approach which uses acceptance and mindfulness strategies, along with commitment and behaviour change strategies, to increase psychological flexibility $[42,43]$. ACT aims to improve work functioning, general skills of psychological flexibility and motivation to return to work despite the presence of pain or other symptoms [43]. Psychological flexibility is described as "the ability to contact the present moment more fully as a conscious human being, and to change or persist in behaviour when doing so serves valued ends" [42, 43]. ACT is increasingly used in various settings and for different patient groups [46] and has shown positive results in several areas [47-51]. A written manual for the ACT sessions (both in groups and individually) has been made by health professionals at the rehabilitation centre in collaboration with an experienced psychologist. The manual describes the intended aims and therapeutic processes of each session, including content 
and techniques. For every session, it also gives examples of observable participant behaviour that could indicate an increase in psychological flexibility. To develop and strengthen the rehabilitations therapists' use of the ACT approach, they receive continuous individual supervision from an experienced psychologist. In addition, visual recordings and observation of therapy sessions are used in individual and group supervision of the therapists, to strengthen fidelity to the approach.

\section{Work-related problem solving}

Work-related problem solving aims to identify challenges and possibilities regarding the return to work process, in order to create an individual return to work plan. Interaction with relevant stakeholders (such as employer, colleagues, GP, welfare case worker or family members), will be discussed, as well as commenced during the rehabilitation process if the participant finds it suitable and helpful. The participant thus chooses whether and how to involve any of these stakeholders. All participants will, in collaboration with their rehabilitation therapist, create their own return to work-plan with individually tailored elements. Here, relevant challenges, measures and solutions regarding the workplace and other parts of the participant's life will be described (for example work adjustments, as well as goals for physical exercise and social life). The employer contributes to the part of the plan which concerns issues at the workplace, and receives this part of the final plan. If the participant consents, the employer receives the whole plan. In addition, the program includes educational sessions on sleep, pain, nutrition and work-related aspects. $\mathrm{ACT}$ is the common ground for and fuels every aspect of the program; an emphasis on developing generalizable and lasting behaviour change that the participants consciously choose in accordance with personal values.

\section{The workplace intervention}

The workplace intervention is inspired by previous successful workplace interventions [7, 16, 52, 53], and was developed by professionals at the rehabilitation center and the research group, with input from the Norwegian labor and welfare administration. The intervention is adapted to the time frame of this rehabilitation program, as well as the geographical, sociocultural and socioeconomic context, also taking into account that these programs will be delivered within a specialist hospital system.

The workplace intervention will consist of a preparatory part during the first two weeks, a meeting at the workplace in the third week of the program, and follow-up work related to the meeting in week four. The individual therapy sessions during week 1 and 2 are used to map the participant's work situation, as well as any potential challenges and possibilities in the return to work process. During the first two weeks of the rehabilitation program the employer is contacted and an appointment for the workplace meeting is made. A one-hour work-specific ACT group session is held during week 2 . This session focuses on the participants' perceptions related to the return to work process, and the possibilities of coping at work despite disabilities are explored.

The participant and the rehabilitation therapists will meet $15-30 \mathrm{~min}$ before the workplace meeting. If it is not agreed upon earlier, they will discuss any limitations of topics which will be discussed with the employer present. A tour on the workplace to view the work arrangements is conducted when found relevant by the participant and rehabilitation therapist. The meeting lasts approximately two hours, and includes the participant, his or her employer, and the participant's rehabilitation therapist. From the employer, the participant's closest leader should always attend, but other persons, such as employee representative or personnel safety representative, considered to be helpful for the return to work process (e.g. due to necessary decision making authority) could also take part. The participant's GP and/or labor and welfare case worker will be informed about the meeting and will be involved when appropriate. The rehabilitation therapist chairs the meeting. The objective is to discuss and plan the participant's return to work process and the actual return. This encompasses identifying the participant and employer's views on any challenges in returning to work, and to discuss potential measures. Conclusions will be made and the responsibility for implementation of any measures will be established.

After the workplace meeting the rehabilitation therapist will contact the employer to ensure that actions agreed upon in the meeting are taken and that measures and solutions will be implemented. A report is sent to those who took part. The participant and the rehabilitation therapist subsequently will discuss the experiences and outcomes from the workplace meeting. The return to work plan is concluded and mailed to the participant's general practitioner. The plan is mailed to the employer if the participant finds it helpful.

\section{Study design}

The study includes three main parts; an effect evaluation, an economical evaluation, and two qualitative studies and one mixed methods study.

\section{Effect and economic evaluation}

The effect evaluation is conducted as a randomized controlled study with a parallel group design. There are three main research questions in the effect evaluation:

- Is an in-patient rehabilitation program with a workplace intervention more effective in increasing return to work (time until full sustainable return to 
work) and reducing sickness absence (total number of sickness absence days during 12 months of follow-up from inclusion), compared to the rehabilitation program only?

- Is an in-patient rehabilitation program with a workplace intervention more effective in improving health, quality of life, and readiness for return to work, compared with the rehabilitation program only?

- Is the in-patient rehabilitation program with a workplace intervention cost- effective, compared to the rehabilitation program only?

\section{Qualitative and mixed methods studies}

Three different studies will be conducted:

- A qualitative interview study will investigate how the rehabilitation therapists attend to the program's main objective - return to work - within a rehabilitation program based on the ACT approach. This study will explore the rehabilitation therapists' experiences with using a novel therapy approach to enhance return to work. (Study A)

- A qualitative interview and observation study will investigate the different stakeholders' (sick-listed person, employer, and rehabilitation therapist's) experiences of the feasibility and outcomes from a workplace intervention. (Study B)

- And a mixed methods study, including quantitative data from questionnaires and qualitative data from interviews, will investigate and elaborate on any changes in the participants' expectations to return to work 4 months after a rehabilitation program with or without a workplace intervention. (Study C)

\section{Data collection}

\section{Effect evaluation}

Data on sickness benefits and other social benefits will be based on register data from the National Welfare Administration. Self-reported data will be collected by internet based questionnaires (www.checkware.com) before screening, before the rehabilitation program starts, right after the rehabilitation program has ended, and 4, 8 and 12 months after the end of the rehabilitation program. Participants are invited by text messages to access the website and answer the questionnaires.

Primary outcomes

- Time from inclusion (i.e. after outpatient screening) until full sustainable return to work (i.e. for at least four weeks without relapse), obtained by national registers.

- Total number of sickness absence days during 12 months of follow-up from inclusion, obtained by national registers.

\section{Secondary outcomes}

- Readiness, beliefs and motivation for return to work (RRTW) [54].

- Expectations regarding future work participation and sick leave:

How long do you think you will be on sick leave from today? (Less than 2 months; 3-6 months; 7-12 months; more than 12 months; I am currently not on sick leave).

Given your current health situation, do you think you will be receiving full sick-leave benefits six months from now? (Yes; no).

Given your current health situation, do you think you will be receiving graded sick leave benefits six months from now? (Yes, less than $50 \%$; yes, $50 \%$ or more).

(The questions were developed during workshops with the research group.)

- Work adjustments and consideration from employer and colleagues:

Has your employer adjusted your work situation according to your health complaints (e.g. changes in work tasks, working hours or facilities)? (Yes; no; I do not need adjustments).

To which extent do you feel that your employer show consideration for your level of functioning? (Very much; to some extent; not much; I do not need special consideration).

To which extent do you feel that your colleagues show consideration for your level of functioning? (Very much; to some extent; not much; I do not need special consideration)

- Job satisfaction:

How do you like your work? (Not at all; not so much; good; very much).

- Return to work self-efficacy (RTWSE-19) [55].

- Health-related quality of life by 15D (15 dimensions) [56].

- Perceived general health:

How is your health now? (Poor; not so good; good; very good.)

- Pain intensity and pain sites by a body pain chart and question 3-5 from the Brief Pain Inventory (BPI) [57].

- Physical activity levels measured by three items concerning frequency, intensity and duration of exercise from the third wave of the HUNT study 
(The HUNT study, Norway. [https://hunt-db. medisin.ntnu.no/hunt-db/\#/instrument/130]).

- Fear-avoidance beliefs about work and physical activity [58].

- Psychological flexibility and acceptance [59].

- Psychological flexibility and acceptance related to work [60].

- Perceived work ability:

How would you rate your current work ability compared with the lifetime best?

(Scale from 0 to 10 , where 10 is best possible work capacity).

- The Hospital Anxiety and Depression Scale (HADS) [61].

\section{Economic evaluation}

Treatment costs for the workplace intervention and the rehabilitation program will be estimated applying a microcosting approach. Productivity costs will be calculated from sickness absence data (days) and age and gender specific wage costs from Statistics Norway. Direct costs in terms of participants' use of health services is recorded by a questionnaire developed and used in a previous study [11]. The questionnaire is answered before the rehabilitation program and 4, 8 and 12 months after the end of the program. It consists of questions concerning the number of:

- days admitted to hospital departments (somatic and psychiatric) or rehabilitation centers

- times receiving medical care (e.g. general practitioner, physiotherapist, chiropractor and occupational care) or alternative treatment (e.g. homeopathy)

- weeks and hours per week of therapeutic work training

- contact-points with the case worker at the The Norwegian Labour and Welfare Service

\section{Qualitative studies}

Data will be collected through individual interviews with participants, rehabilitations therapists and employers, and through participant observation of the rehabilitation programs and non-participant observation of the workplace intervention. All interviews will be semi-structured and based on an interview guide. Interviews will be audiorecorded and transcribed. Data from observation will be collected through written field notes.

Study A: Therapists will be interviewed individually to investigate how they attend to the return to work process within a rehabilitation program based on ACT. Interviews will take part when the therapists have gained some experience with the program and the intervention. The main topics in the interviews will be what the therapists perceive as the main aim and potential outcomes of the program, and how they attend to the participants' return to work process during their work. Participant observation of the rehabilitation program will be conducted and will be added to the interviews as data material.

Study B: Approximately 10-15 of the workplace interventions will be followed with non-participant observation. Stakeholders taking part in the workplace intervention (participants, employers and rehabilitation therapists) will be interviewed after the workplace meeting to investigate their experiences. The main topics in the interviews will be the usability and potential outcomes of the current intervention, and how the intervention might be improved. We will aim to include triads with participants, employers and rehabilitation therapists who have attended the same workplace meeting.

Study C: Participants will be interviewed to explore their perceptions of the return to work process four months after the end of the rehabilitation program. Interview topics will be the participants' experiences of their current work participation and expectations towards the return to work process. Data from interviews will be combined with findings from quantitative data on job satisfaction, return to work self-efficacy, and readiness, expectations, beliefs and motivation for return to work.

\section{Data analyses \\ Effect evaluation}

Effect analyses of primary and secondary outcomes will be performed by intention to treat and per protocol. For the primary outcomes, sustainable return to work will be evaluated with survival analysis and difference in days of sick leave will be evaluated with the Mann Whitney Utest, as sick leave days are not likely to be normally distributed. Effect differences for secondary outcomes will be analysed with linear mixed models or non-parametric methods, as appropriate. Subgroup analyses will be performed for main diagnosis groups.

\section{Economic evaluation}

We will take the societal perspective and both a costeffectiveness, cost-utility and cost benefit approach will be applied, and in accordance with the Norwegian guidelines for economic evaluation [62]. In the cost-effectiveness analysis, sickness absence days will be used as outcome measure, and to avoid double counting productivity costs will be excluded. In the cost-utility analysis, the outcome measure is Quality Adjusted Life Years [63] based on the 15D instrument [56], and cost-utility estimates will be provided both with and without the inclusion of productivity costs. The cost-benefit analysis will compare costs and benefits taking increased treatment costs and potential societal savings from reduced sickness absenteeism into account. Analyses of cost-effectiveness and cost-utility will be performed by 
calculating the ratio of incremental costs divided by incremental effect. Univariate sensitivity analyses and bootstrapping procedures will be applied to estimate uncertainty surrounding the incremental ratios.

\section{Qualitative studies}

Analysis of interviews will be inspired by interpretive phenomenological analysis (IPA) [64], while analysis of the observational studies will be inspired by thematic analysis [65].

\section{Ethics}

The Regional Committee for Medical and Health Research Ethics in Central Norway has approved the protocol (No.: 2014/2279), and the trial is registered in clinicaltrials.gov (NCT02541890). All participants who are included in qualitative observation and interview studies will sign an informed consent before they take part in the study.

\section{Discussion}

As described in the Background, designs suitable to encompass complex and novel interventions provided to a heterogeneous patient group are wanting. Researchers have emphasized the need for good quality evaluations of initiatives intended to enhance return to work [66]. The study described in this protocol article will combine an effect evaluation, economic evaluations, and qualitative and mixed methods study in a comprehensive approach.

Although different countries have different arrangements for follow-up and benefits during sickness absence, the results from this study could be useful for contexts similar to the Norwegian. The rehabilitation program provided in this study takes place in an in-patient rehabilitation center setting, but is not dependent on this setting to be replicated. Any results regarding effect on return to work or health outcomes should thus be generalizable to other contexts. Since the intervention takes place at the participant's workplace, it would be useful for other contexts, and the intervention in itself is applicable in most workplace settings. The design of this study will make it possible to assess effects of the program, both regarding work participation and economic benefit. In addition to providing results in its own right, qualitative studies nested in randomized controlled trials are recommended as a way to enhance the significance of the results [67-69]. The qualitative results could also be helpful in modifying and applying the different parts of the rehabilitation program and the workplace meeting into other contexts.

The heterogeneous patient group as well as the complexity of the rehabilitation program and the workplace intervention, are all aspects which make a good and robust study design very important. Adding the patients' perspectives in qualitative studies might give valuable insight for the development of further studies. The exploration of the different stakeholders' perspectives and experiences with the same workplace intervention could provide new and interesting knowledge on the suitability of such interventions, as well as any barriers which are not accounted for in the development of the intervention. Input from patients and employers will be very important to make necessary improvements.

Although the researchers are blinded during the analyses, this study's randomized controlled design does not allow for any blinding of the participants or rehabilitation therapists. The rehabilitation programs in this study are designed according to the Norwegian systems and culture regarding benefits, work, health, and policies. The results from the study are thus not necessarily generalizable to countries with different socio-economic and socio-cultural contexts than Norway. To implement similar rehabilitation programs in other countries necessary modifications should be considered.

\section{Impact of results}

Investigating the stakeholders' experiences and evaluations are necessary supplements to studies investigating the quantitative effects from an intervention. The combination of quantitative and qualitative data will provide knowledge on important individual and contextual causal and predictive factors during the return to work process. Professionals providing health and work services will benefit from the results in how to best support sicklisted persons to return to work. The results on effect and cost-effectiveness generated in this study will give policy makers a knowledge base for making decisions for future policies in this field.

\section{Acknowledgements \\ Not applicable.}

\section{Funding}

This study received allocated government funding through the Central Norway Regional Health Authority, and is funded by a grant to MBR, LA and MSF by The Research Council of Norway. The funding body had no role in either the design of the study or in the writing of the manuscript, and will not have any role in data collection or analyses.

\section{Availability of data and materials}

Not applicable.

\section{Authors' contributions}

MBR was in charge of writing and completing the manuscript. MS, HT, TNJ, $P B, R J, M B R, M S F, C J, L A$ and SBL participated in designing one or more parts of the complex rehabilitation program and/or the workplace intervention. NEK is conducting the qualitative studies and participated in the design of these. $\mathrm{VH}$ is mainly responsible for and wrote the section on the economic evaluation study. All authors participated in completion of and approved the final manuscript.

\section{Ethics approval and consent to participate}

The Regional Committee for Medical and Health Research Ethics in Central Norway has approved the protocol (No.: 2014/2279), and the trial is registered in clinicaltrials.gov (NCT02541890). All participants who are included in qualitative observation and interview studies will sign an informed consent before they take part in the study. 


\section{Consent for publication}

Not applicable.

\section{Competing interests}

MBR participated in 2017 and 2018 as a research advisor regarding a study conducted and funded by Janssen-Cilag A/S. All other authors declare that they have no competing interests.

\section{Publisher's Note}

Springer Nature remains neutral with regard to jurisdictional claims in published maps and institutional affiliations.

\section{Author details \\ ${ }^{1}$ Department of Public Health and Nursing, Norwegian University of Science and Technology, Trondheim, Norway. ${ }^{2}$ Department of Mental Health, Norwegian University of Science and Technology, 7491 Trondheim, Norway ${ }^{3}$ Department of Physical Medicine and Rehabilitation, St. Olav's Hospital, Trondheim University Hospital, Trondheim, Norway. ${ }^{4}$ Hysnes Rehabilitation Center, Trondheim University Hospital, Trondheim, Norway. ${ }^{5}$ Department of Circulation and Medical Imaging, Faculty of Medicine, Norwegian University of Science and Technology, Trondheim, Norway. ${ }^{6}$ National Centre for Occupational Rehabilitation, Rauland, Norway.}

\section{Received: 16 May 2017 Accepted: 30 January 2018}

\section{Published online: 05 February 2018}

\section{References}

1. OECD. Sickness, disability and work: Breaking the barriers. A synthesis of findings across OECD countries. Paris: OECD publishing; 2010.

2. OECD. Reconsidering Norwegian sickness absence policies. In: Mental health and work. Paris: OECD Publishing; 2013.

3. Staal B, deRijk A, Houkes I, Heymans M. Clinical interventions to reduce work disability in workers with musculoskeletal disorders or mental health problems. In: Loisel P, Anema J, editors. In Handbook of work disability Prevention and management. New York: Springer; 2013. p. 317-34.

4. Poulsen OM, Aust B, Bjorner JB, Rugulies R, Hansen JV, Tverborgvik T, et al. Effect of the Danish return-to-work program on long-term sickness absence: results from a randomized controlled trial in three municipalities. Scand J Work Environ Health. 2014;40:47-56.

5. Oyeflaten I, Hysing M, Eriksen HR. Prognostic factors associated with return to work following multidisciplinary vocational rehabilitation. J Rehabil Med. 2008;40:548-54.

6. Andersen LN, Juul-Kristensen B, Roessler KK, Herborg LG, Sorensen TL, Sogaard K. Efficacy of 'Tailored physical Activity' or 'Chronic pain selfmanagement Program' on return to work for sick-listed citizens: design of a randomised controlled trial. BMC Public Health. 2013;13:66.

7. Lambeek LC, van MW KDL, Loisel P, Anema JR. Randomised controlled trial of integrated care to reduce disability from chronic low back pain in working and private life. BMJ. 2010;340:C1035.

8. Reme SE, Grasdal AL, Lovvik C, Lie SA, Overland S. Work-focused cognitivebehavioural therapy and individual job support to increase work participation in common mental disorders: a randomised controlled multicentre trial. Occup Environ Med. 2015;72:745-52.

9. Myhre K, Marchand GH, Leivseth G, Keller A, Bautz-Holter E, Sandvik L, et al. The effect of work-focused rehabilitation among patients with neck and back pain: a randomized controlled trial. Spine (Phila Pa 1976 ). 2014;39: 1999-2006.

10. Braathen TN, Veiersted KB, Heggenes J. Improved work ability and return to work following vocational multidisciplinary rehabilitation of subjects on long-term sick leave. J Rehabil Med. 2007;39:493-9.

11. Fimland MS, Vasseljen O, Gismervik S, Rise MB, Halsteinli V, Jacobsen HB, et al. Occupational rehabilitation programs for musculoskeletal pain and common mental health disorders: study protocol of a randomized controlled trial. BMC Public Health. 2014;14:368.

12. Aasdahl L, Pape K, Vasseljen O, Johnsen R, Gismervik S, Jensen C, et al. Effects of inpatient multicomponent occupational rehabilitation versus less comprehensive outpatient rehabilitation on somatic and mental health: secondary outcomes of a randomized clinical trial. J Occup Rehabil. 2016;

13. Econ Povry. [occupational rehabilitation. Report for the Norwegian Directorate for Work and Welfare]. R-2010-057. 2010. Oslo.
14. Vermeulen SJ, Anema JR, Schellart AJ, Knol DL, van MW, van $\operatorname{der}$ Beek AJ. A participatory return-to-work intervention for temporary agency workers and unemployed workers sick-listed due to musculoskeletal disorders: results of a randomized controlled trial. J Occup Rehabil. 2011;

15. Jensen C, Jensen OK, Christiansen DH, Nielsen CV. One-year follow-up in employees sick-listed because of low back pain: randomized clinical trial comparing multidisciplinary and brief intervention. Spine (Phila Pa 1976 ). 2011;36:1180-9.

16. Loisel P, Abenhaim L, Durand P, Esdaile JM, Suissa S, Gosselin L, et al. A population-based, randomized clinical trial on back pain management. Spine (Phila Pa 1976 ). 1997;22:2911-8.

17. Hagen EM, Eriksen HR, Ursin H. Does early intervention with a light mobilization program reduce long-term sick leave for low back pain? Spine (Phila Pa 1976 ). 2000;25:1973-6.

18. Blonk R, Brennikmeijer V, Lagerveld S, Houtman I. Return to work: a comparison of two cognitive behavioural interventions in cases of work-related psychological complaints among the self-employed. Work \& Stress. 2006;20:129-44.

19. van Oostrom SH, van MW, Terluin B, de Vet HC, Knol DL, Anema JR. A workplace intervention for sick-listed employees with distress: results of a randomised controlled trial. Occup Environ Med. 2010;67:596-602.

20. van der Klink JJ, Blonk RW, Schene AH, van Dijk FJ. Reducing long term sickness absence by an activating intervention in adjustment disorders: a cluster randomised controlled design. Occup Environ Med. 2003;60:429-37.

21. Netterstrom B, Bech P. Effect of a multidisciplinary stress treatment programme on the return to work rate for persons with work-related stress. A non-randomized controlled study from a stress clinic. BMC Public Health. 2010;10:658.

22. Costa-Black K, Feuerstein M, Loisel P. Work disability models: past and present. In: Loisel P, Toronto AJ, editors. Handbook of Work Disability Prevention and Management. New York: Springer; 2013. p. 71-93.

23. Briand C, Durand MJ, St-Arnaud L, Corbiere M. Work and mental health: learning from return-to-work rehabilitation programs designed for workers with musculoskeletal disorders. Int J Law Psychiatry. 2007;30:444-57.

24. Hagen EM, Svensen E, Eriksen HR, Ihlebaek CM, Ursin H. Comorbid subjective health complaints in low back pain. Spine (Phila Pa 1976 ). 2006; 31:1491-5.

25. Reme SE, Tangen T, Moe T, Eriksen HR. Prevalence of psychiatric disorders in sick listed chronic low back pain patients. Eur J Pain. 2011;15:1075-80.

26. Natvig B, Eriksen W, Bruusgaard D. Low back pain as a predictor of longterm work disability. Scand J Public Health. 2002;30:288-92.

27. Von KM, Crane P, Lane M, Miglioretti DL, Simon G, Saunders K, et al. Chronic spinal pain and physical-mental comorbidity in the United States: results from the national comorbidity survey replication. Pain. 2005;113:331-9.

28. Anema J, Loisel P. Handbook of work disability. Prevention and management. New York: Springer; 2013.

29. Desiron HA, Crutzen R, Godderis L, Van HE, de RA. Bridging health care and the workplace: formulation of a return-to-work intervention for breast cancer patients using an intervention mapping approach. J Occup Rehabil. 2016;26:350-65.

30. Hoefsmit N, Houkes I, Nijhuis FJ. Intervention characteristics that facilitate return to work after sickness absence: a systematic literature review. J Occup Rehabil. 2012;22:462-77.

31. Kuoppala J, Lamminpaa A. Rehabilitation and work ability: a systematic literature review. J Rehabil Med. 2008;40:796-804.

32. van Vilsteren $\mathrm{M}$, van Oostrom $\mathrm{SH}$, de Vet $\mathrm{HC}$, Franche $\mathrm{RL}$, Boot $\mathrm{CR}$, Anema JR. Workplace interventions to prevent work disability in workers on sick leave. Cochrane Database Syst Rev. 2015:CD006955.

33. Young A. Return to work stakeholders' perspectives on work disability. In: Handbook of work disability. Prevention and management. New York: Springer; 2013. p. 409-23.

34. Schandelmaier S, Ebrahim S, Burkhardt SC, de Boer WE, Zumbrunn T, Guyatt $\mathrm{GH}$, et al. Return to work coordination programmes for work disability: a meta-analysis of randomised controlled trials. PLoS One. 2012;7:e49760.

35. Bultmann U, Sherson D, Olsen J, Hansen CL, Lund T, Kilsgaard J. Coordinated and tailored work rehabilitation: a randomized controlled trial with economic evaluation undertaken with workers on sick leave due to musculoskeletal disorders. J Occup Rehabil. 2009;19:81-93.

36. Anema JR, Steenstra IA, Bongers PM, de Vet HC, Knol DL, Loisel P, et al. Multidisciplinary rehabilitation for subacute low back pain: graded activity or workplace intervention or both? A randomized controlled trial. Spine (Phila Pa 1976 ). 2007;32:291-8. 
37. Palmer KT, Harris EC, Linaker C, Barker M, Lawrence W, Cooper C, et al. Effectiveness of community- and workplace-based interventions to manage musculoskeletal-related sickness absence and job loss: a systematic review. Rheumatology (Oxford). 2012:51:230-42.

38. Carroll C, Rick J, Pilgrim H, Cameron J, Hillage J. Workplace involvement improves return to work rates among employees with back pain on longterm sick leave: a systematic review of the effectiveness and costeffectiveness of interventions. Disabil Rehabil. 2010;32:607-21.

39. van Oostrom S, Boot R. Workplace interventions. In: Loisel P, Anema JR, editors. Handbook of Work Disability Prevention and Management. London: Springer; 2013. p. 335-55.

40. Aasdahl L, Pape K, Vasseljen O, Johnsen R, Gismervik S, Halsteinli V, et al. Effect of inpatient multicomponent occupational rehabilitation versus less comprehensive outpatient rehabilitation on sickness absence in persons with musculoskeletal- or mental health disorders: a randomized clinical trial. J Occup Rehabil. 2017;

41. Rise MB, Gismervik SO, Johnsen R, Fimland MS. Sick-listed persons' experiences with taking part in an in-patient occupational rehabilitation program based on acceptance and commitment therapy: a qualitative focus group interview study. BMC Health Serv Res. 2015;15:526.

42. Hayes SC, Strosahl K, Wilson KG. Acceptance and Commptment therapy: an experiential appriach to behavior change. New York: Guilford Press; 1999.

43. Hayes SC, Luoma JB, Bond FW, Masuda A, Lillis J. Acceptance and commitment therapy: model, processes and outcomes. Behav Res Ther. 2006;44:1-25.

44. Dahl J, Wilson KG, Nilsson A. Acceptance and commitment therapy and the treatment of persons at risk for long-term disability resulting from stress and pain symptoms: a preliminary randomized trial. Behav Ther. 2004;35:785-801.

45. Lytsy P, Carlsson L, Anderzen I. Effectiveness of two vocational rehabilitation programmes in women with long-term sick leave due to pain syndrome or mental illness: 1-year follow-up of a randomized controlled trial. J Rehabil Med. 2017:49:170-7.

46. Hayes SC, Levin ME, Plumb-Vilardaga J, Villatte JL, Pistorello J. Acceptance and commitment therapy and contextual behavioral science: examining the progress of a distinctive model of behavioral and cognitive therapy. Behav Ther. 2013:44:180-98.

47. Tjak JG, Davis ML, Morina N, Powers MB, Smits JA, Emmelkamp PM. A metaanalysis of the efficacy of acceptance and commitment therapy for clinically relevant mental and physical health problems. Psychother Psychosom. 2015; 84:30-6.

48. Pull CB. Current empirical status of acceptance and commitment therapy. Curr Opin Psychiatry. 2009;22:55-60.

49. Yovel I. Acceptance and commitment therapy and the new generation of cognitive behavioral treatments. Isr J Psychiatry Relat Sci. 2009:46:304-9.

50. Bailey KM, Carleton RN, Vlaeyen JW, Asmundson GJ. Treatments addressing pain-related fear and anxiety in patients with chronic musculoskeletal pain: a preliminary review. Cogn Behav Ther. 2010;39:46-63.

51. Powers MB, MB ZVSV, Emmelkamp PM. Acceptance and commitment therapy: a meta-analytic review. Psychother Psychosom. 2009;78:73-80.

52. van Oostrom SH, Driessen MT, de Vet HC, Franche RL, Schonstein E, Loisel P, et al. Workplace interventions for preventing work disability. Cochrane Database Syst Rev. 2009:CD006955.

53. van Oostrom S, Boot C. Workplace interventions. In: Handbook of work disability Prevention and management. New York: Springer; 2013. p. 335-55.

54. Franche RL, Corbiere M, Lee H, Breslin FC, Hepburn CG. The readiness for return-to-work (RRTW) scale: development and validation of a self-report staging scale in lost-time claimants with musculoskeletal disorders. J Occup Rehabil. 2007;17:450-72.

55. Brouwer S, Franche RL, Hogg-Johnson S, Lee H, Krause N, Shaw WS. Returnto-work self-efficacy: development and validation of a scale in claimants with musculoskeletal disorders. J Occup Rehabil. 2011;21:244-58.

56. Sintonen $\mathrm{H}$. The $15 \mathrm{D}$ instrument of health-related quality of life: properties and applications. Ann Med. 2001;33:328-36.

57. Klepstad P, Loge JH, Borchgrevink PC, Mendoza TR, Cleeland CS, Kaasa S. The Norwegian brief pain inventory questionnaire: Translation and validation in cancer pain patients. J Pain Symptom Manage. 2002;24:517-25.

58. Waddell G, Newton M, Henderson I, Somerville D, Main CJ. A FearAvoidance Beliefs Questionnaire (FABQ) and the role of fear-avoidance beliefs in chronic low back pain and disability. Pain. 1993;52:157-68.

59. Bond FW, Hayes SC, Baer RA, Carpenter KM, Guenole N, Orcutt HK, et al. Preliminary psychometric properties of the acceptance and action
questionnaire-II: a revised measure of psychological inflexibility and experiential avoidance. Behav Ther. 2011;42:676-88.

60. Bond FW, Lloyd J, Guenole N. The work-related acceptance and action questionnaire: initial psychometric findings and their implications for measuring psychological flexibility in specific contexts. J Occup Organ Psychol. 2013;86:331-47.

61. Zigmond AS, Snaith RP. The hospital anxiety and depression scale. Acta Psychiatr Scand. 1983;67:361-70.

62. Norwegian Directorate of Health. Økonomisk evaluering av helsetiltak enveileder [Economic evaluation of health services - a guide]. 2012.

63. Drummond MF, Schulpher MJ, Torrance GW, O'Brian BJ, Staddard GL. Standard methods for the economic evaluation of health care programmes. Third ed. Oxford: Oxford University Press; 2005.

64. Smith JA, Flower P, Larkin M. Interpretative phenomenological analysis: theory, method and research. London: Sage; 2009.

65. Howitt D. Introduction to Qualitative Methods in Psychology. 2nd edn. Pearson Education Limited; 2012.

66. Noben CY, Nijhuis FJ, de Rijk AE, Evers SM. Design of a trial-based economic evaluation on the cost-effectiveness of employability interventions among work disabled employees or employees at risk of work disability: the CASEstudy. BMC Public Health. 2012;12:43.

67. Bradley F, Wiles R, Kinmonth AL, Mant D, Gantley M. Development and evaluation of complex interventions in health services research: case study of the Southampton heart integrated care project (SHIP). The SHIP collaborative group. BMJ. 1999;318:711-5.

68. Lewin S, Glenton C, Oxman AD. Use of qualitative methods alongside randomised controlled trials of complex healthcare interventions: methodological study. BMJ. 2009;339:b3496.

69. Campbell M, Fitzpatrick R, Haines A, Kinmonth AL, Sandercock P, Spiegelhalter $D$, et al. Framework for design and evaluation of complex interventions to improve health. BMJ. 2000;321:694-6.

\section{Submit your next manuscript to BioMed Central and we will help you at every step:}

- We accept pre-submission inquiries

- Our selector tool helps you to find the most relevant journal

- We provide round the clock customer support

- Convenient online submission

- Thorough peer review

- Inclusion in PubMed and all major indexing services

- Maximum visibility for your research

Submit your manuscript at www.biomedcentral.com/submit
Biomed Central 